Journal of Advanced Computer Science \& Technology, 8 (1) (2019) $11-15$
Journal of Advanced Computer Science \& Technology
SPC
Website: www.sciencepubco.com/index.php/JACST
Research paper

\title{
Subarea tree routing algorithm based infrastructure for mobile ad-hoc networks
}

\author{
Omeed Kamal Khorsheed ${ }^{1}$, Prof Dr Abdul Hanan Abdullah ${ }^{1}$ \\ ${ }^{1}$ University of technology Malaysia, Facility of computing \\ *Corresponding author E-mail: Omeed.kamal-1984@graduate.utm
}

\begin{abstract}
Ad hoc networks are self-organizing and multi-hop networks for data communication. Subarea Tree Routing (STR) has used for multihop networks for network division into various subareas. The main objective of this paper is creating a dynamic sub-area tree infrastructure for Mobile Ad hoc Networks (MANET). The proposed architecture uses the hierarchical procedure to divide the whole network into many geographical sub-area networks. In addition, each sub-area has a selected root node, we can configure the root node manually due to small ad hoc network and uses auto-discovery procedure to select the roots for large networks. The root node is located at the center of sub-area with hop level zero. The root function is used to update the routing table and root table for sub-area. After sub-area created any interconnected node detects a new inefficient node for sending the invitation message to join the sub-area tree. In the same sub-area, proactive routing protocols are used between the sub-area root node and its interconnect nodes and reactive routing protocols are used between all sub-areas root nodes. The new sub-area tree provides an infrastructure to avoid the flooding procedures. Routing in the subarea tree avoids the transmission collision to optimize the ad-hoc networks.
\end{abstract}

Keywords: STR; Clustering; Sub-area; Multi-Hop; Mobility; Tree; Routing Table; Proactive; Reactive.

\section{Introduction}

Wireless Ad-hoc networks are self-organized networks where all the devices are communicating for different purposes. The devices in these networks do not rely on pre-existing infrastructure or centralized control devices [1]. The devices in ad hoc networks are static or mobile and changing their topologies based on area. In these networks, every device acts as a router and has its own transmission range to communicate with neighbor nodes. Due to heterogeneous, multi-hop and various devices, these networks have suffered from various routing challenges. Data communication between two nodes are not always possible and can be based on multi-hop networking. The routes for data routing are not stable due to various obstacles, interference, and mobility of devices. Most of the routing protocols have been designed to provide stable paths between the source and destination nodes. Due to infrastructure fewer networks, these networks require a formidable work to implement appropriate routing protocols for data routing for mobile ad-hoc networks topologies. In addition, the designed protocols should be able to handle the limited bandwidth, energy and unstable connections between the sender and receiver nodes in the network. Another challenge is to handle the high probability of nodes failure or disappearing from the communication range and cause of disconnection and delay issues [2].

In order to address the routing challenges in ad hoc networks, the broadly designed protocols are categorized into two categories proactive or table-driven routing protocols and reactive or on-demand routing protocols [3]. Proactive and reactive protocols have used to achieve the distributed communications between nodes without paying attention to the dynamic infrastructure of MANET [4]. Some other routing protocols have developed to support routing in the dynamic infrastructure like flat routing protocols and hierarchical routing protocols [5], [6].

Flat routing protocols have able to distribute the routing information to all the nodes which are connected to each other as peers without any segmentation or organizing structure. These protocols enable to deliver the data packets among routers through any available path without considering network hierarchy, distribution, and energy composition. In hierarchical routing protocols, router nodes are categorized into groups known as sub-areas. Each router node has only the information about the nodes in its own subarea instead of information about other nodes in other sub areas. In this paper, the proposed work is based on a hierarchical routing protocol with the feature of Sub-Area Tree Routing (STR) mechanism. In this method, the network is divided into many sub-areas for better scalability and reduces the delay and overhead in the multi-hop networks. The proposed protocol includes the main advantage of a reactive protocol to reduce the routing overhead. It also uses the proactive method as an advantage to reduce the routing delay.

The rest of the paper is organized as follows: Section 2 presents the related work. Section 3 discusses the proposed routing protocol design and routing process. Section 4 presents the performance analysis of the proposed protocol. In last, the paper concludes with future direction. 


\section{Related work}

In this section, the related work discusses on MANET. As discussed, MANET is working without any pre-existing infrastructure. In this context, various studies have designed dynamic infrastructure for these networks. A Spanning Tree Routing Protocol [7] was proposed to provide a dynamic topology as an infrastructure for data communication. This protocol main objective is to avoid the flooding issues in routing.

Gui-kai, et al. [8] was proposed a Subarea Tree Routing for Multi-hop Ad-hoc Networks. This protocol adopts the subarea method in autonomous reconfiguration system to avoid the delay in data transmission in MANET. The main objective of this study is to creating sub-area trees which are depending on the environment of the ad-hoc network. Afterward, the whole network is divided into many subareas. STR protocol consists of two types of the node including root and interconnects nodes. This protocol uses two methods namely proactive and reactive. With the help of a proactive strategy, this protocol established the routing between root and interconnect nodes. On the other hand, the reactive protocol has used between internal and subarea nodes.

Another protocol in [9], proposed a clustering approach to enhance the MANET networks. This approach simplifies the routing processes in the network by adopting the weight-based clustering mechanism. This protocol forms clusters and cluster head to provide more stable and flexible networks. This protocol adopts various parameters to form a weight function. However, the clustering approach has more overhead where the sensor nodes always searching for cluster head and forming the clusters in the network.

Proactive routing protocols have designed to overcome the routing issues by using updated routing information and create the concurrently routing table for MANET [10]. In these protocols, the nodes are broadcasting the periodically update message to all the neighbor nodes. On the other hand, the reactive type [11] protocols are used to find the transmission route or path on demand just when node start sending a data packet to another receiver node in the same network. These types of protocols are working by flooding the route request packets in the network. When the sender node decides to connect with the destination node it will directly send the route request packet as a broadcast message to all direct neighbors in the first level. If the destination node found in the first level and received the router request it will respond with a replay packet. But if the destination node is not in the first level then the neighbor's nodes in the first level will directly forward the broadcast router request packet to their neighbors in the level 2 until the destination node is found and respond with a replay message to the sender node in the shortest transmission path.

Pandey and Biswas [12] was proposed a proactive approach to form a binary tree structure by decomposing large network to subnetworks. The subnetworks are monitored by using an agent node which is selected through several broadcasted regulations. Agent node is working as a facilitator to maintain the routing information for local and other agent's nodes. The network is divided into equal independent sub-networks and then follow up to a feasible number of nodes in each subnetwork. One another study [13] was proposed a routing protocol based on binary-tree shaped network. This protocol derives from the routing table driven method for MANET. This is one demand routing protocol to maintain the network topologies and find reliable and the suitable path between nodes. This protocol minimizes the total number of control packets and enables them to decrease data collision rate. In addition, this protocol also establishes the binary tree with a root node and working as a gateway node.

Another approach to constructing the network topology is mesh networking, where all network member nodes are working together for data transmission and its distribution in the network [14]. The data transmission in mesh topology is based on either a flooding mechanism or using routing mechanism. In this type, source nodes broadcast a multi-hop message to all mesh nodes until it reaches to the destination node. Mesh network try to preserve the availability of the transmission path by reconfiguring its topology in order to repair a broken path. This strategy is used in self-healing protocols like (Shortest Path Bridging) to operate the network when some nodes fall or when the transmission path becomes unavailable. Mesh networks are considered as a type of MANET which reduces the mobility problem where nodes are moving out from the range of the network.

\section{Proposed sub tree routing}

In this paper, we proposed a sub-area tree routing algorithm. The main objective of the proposed protocol is to create a virtual hierarchical infrastructure (depend on time and nodes mobility) for multi-hop networks. In addition, the proposed algorithm creates a hierarchical mesh network or hierarchical spanning tree network which include multi sub-tree where each tree represents a sub-area network with one root node.

\subsection{Proposed algorithm procedure}

Proposed algorithm has three main steps:

Step 1: Select the root nodes for each sub-area.

Step 2: Invite the inefficient neighbor nodes to join the sub-area.

Step 3: Permanence the network tree topology.

Step 1: Select the Root Nodes for each Sub-Area

In this step, we assume that all nodes are in a geographical area. Every single node is in the autonomous mode with a unique ID.

In the start, we are looking for a root node in the center of sub-area. The root node is in the hop level zero of sub-area. Then, we can identify the root node manually by selecting the most efficient node or we can use the complex process auto-discovery to select the root node. This strategy has adopted for selecting the minimum weight node for Cluster Head $(\mathrm{CH})$ node.

Naturally, we deal with a mobile node which can move from the sub-area or maybe out from the range at any time and for any reason. The manual method is only used for small networks.

Auto-discovery procedure needs to determine the root arrangement like:

- $\quad$ Node ID.

- Node Power Level.

- Transmission range.

- Count of neighbor nodes in the transmission range.

- Hop Level.

Those arrangements formed the Discovery Request Message (DRM) which broadcasts by any activated node in the sub-area at any time (T). Then, any node in the sub-area received the request message and compatible with the root arrangement which is considered as a root node. 
A root node of sub-area can use a proactive method to communicate with its interconnect nodes and update the routing table or routing list for all route nodes in the sub-area. In the same time, the root node broadcasts its ID and routing list to all root nodes. Therefore, all root nodes have information about the roots of the network. The communication between root nodes is possible through a reactive routing protocol.

As a result of this step, we have two kinds of tables called routing table of sub-area and roots table with their routers at $\mathrm{T}$ time. The routing table of sub-area is showing in below Table 1.

Table 1: Sub Area Routing Table

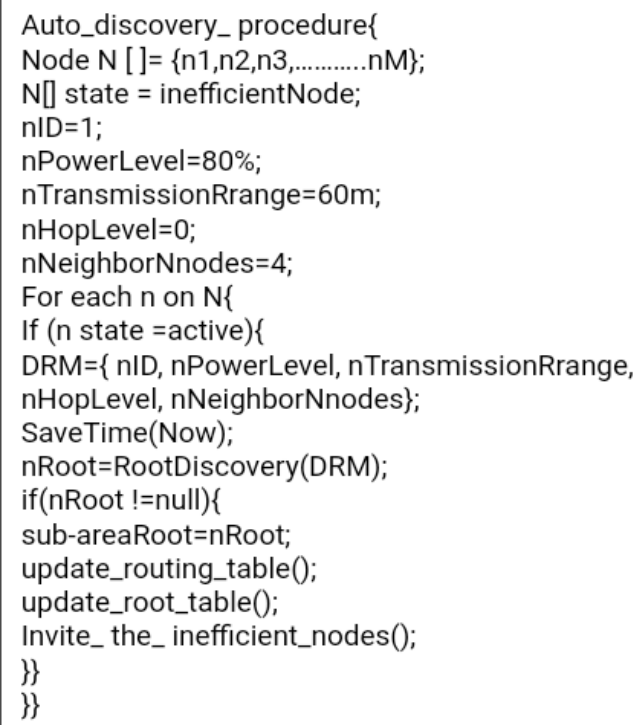

The below Figures 1, 2 and 3 present the root selection process in the network.

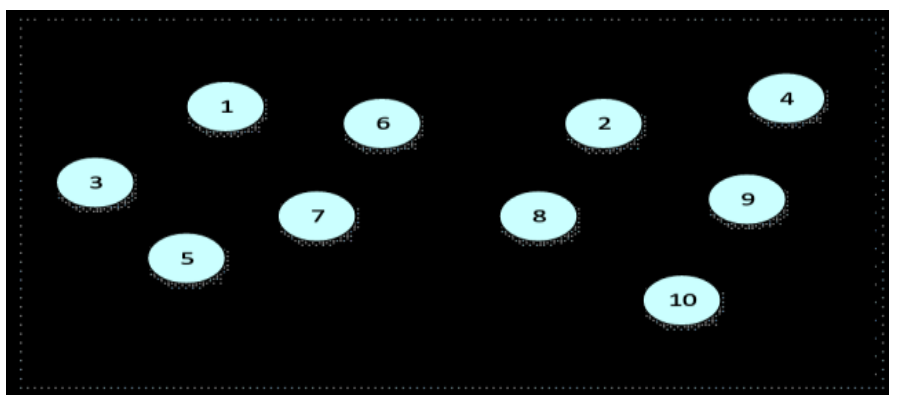

Fig. 1: Inefficient Nodes in a Geographical Area.

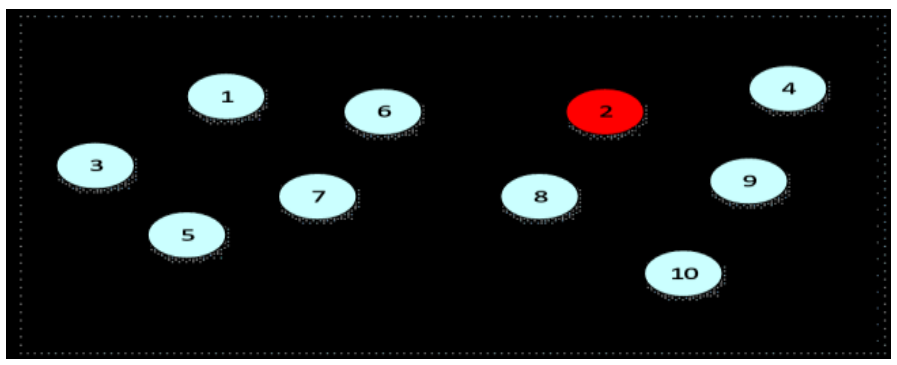

Fig. 2: Node 2 Activated at Time T and send a DRM.

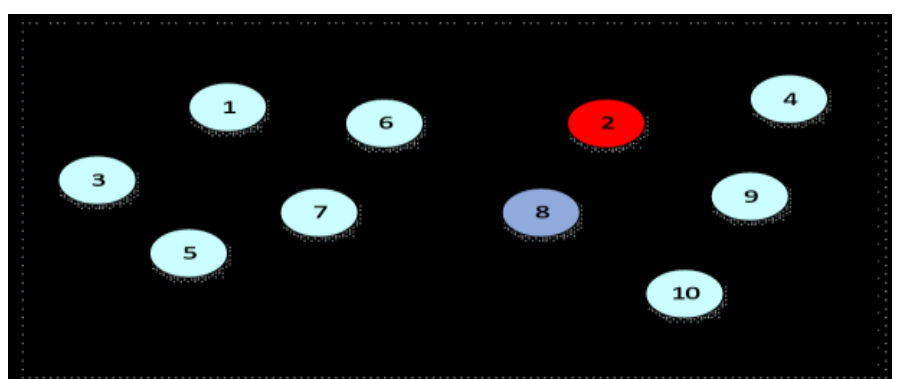

Fig. 3: Node 8 Compatible with the Root Arrangement and Considered as Root Node.

Step 2: Invite the Inefficient Neighbor Nodes to Join the Sub-area 
At any time (T2), a tree node detected a new inefficient node in the neighborhood immediately. Then it sends the invitation message and broadcasts a detection message including the detected node ID and the Time T2. The inefficient node will be active and join sub-area tree. The root node will update its routing table to inform others root about the new tree node as shown in Table 2.

Table 2: Join the Sub Area Table
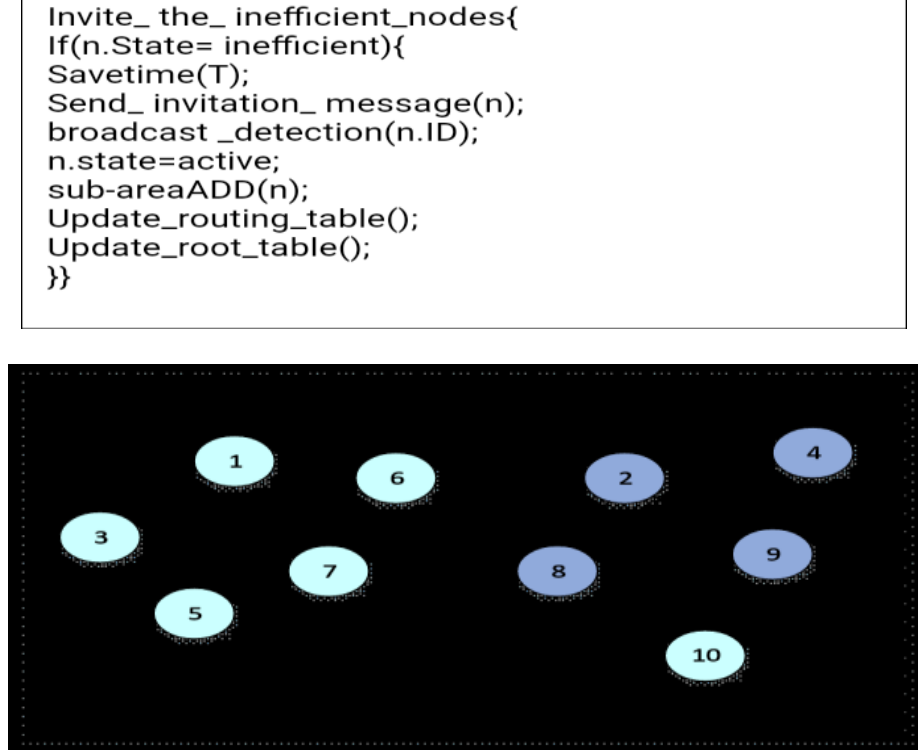

Fig. 4: Sub-Area Nodes 2,4,8,9 and the Root Is 8

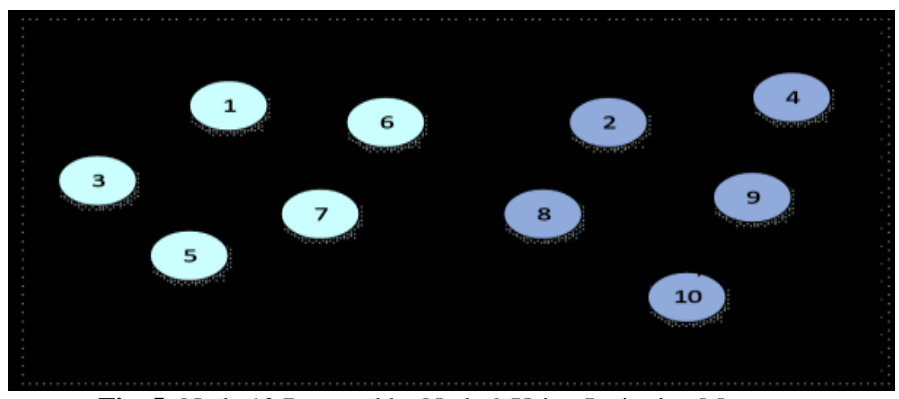

Fig. 5: Node 10 Detected by Node 9 Using Invitation Message.

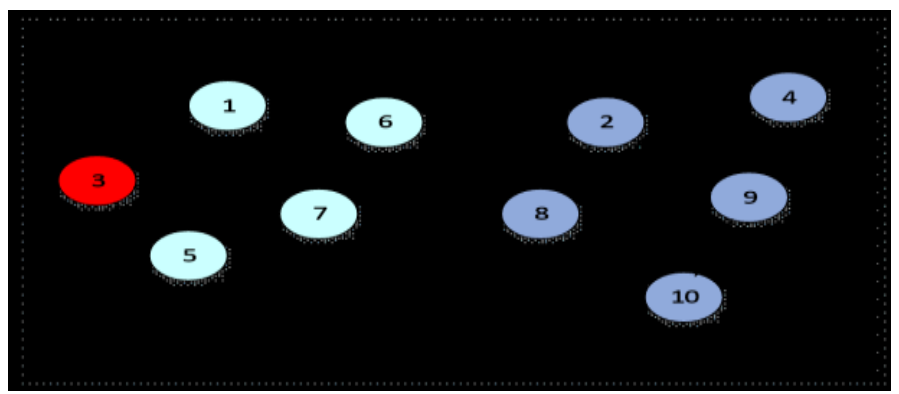

Fig. 6: Node 3 Activated at Time T2 and Send A DRM.

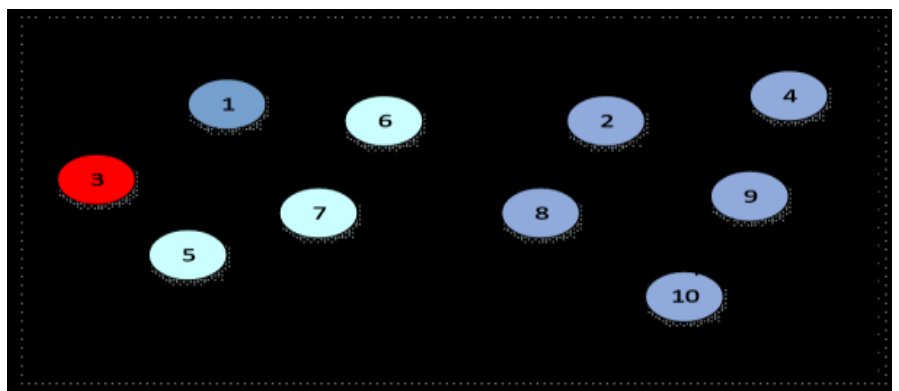

Fig. 7: Node 1 Compatible with the Root Arrangement and Considered as Root Node. 


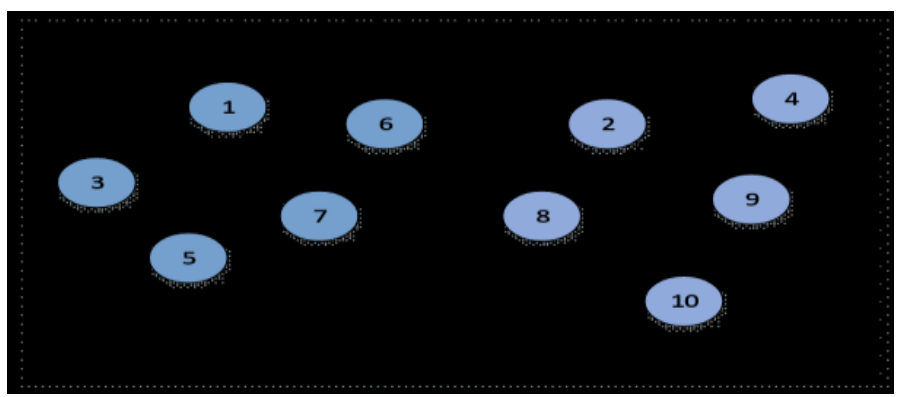

Fig. 8: Sub-Area Tree Topology.

Step 3: Permanence the Network Tree Topology

In this step, we try to keep the permanence of tree topology and we are facing:

- A tree node departed the sub-area network: when a tree node departed from the sub-area in the network then the root node informs its tree nodes about the departure node and updates its routing table.

- A root node departed the tree network: when a root node departed the tree network it will effect on all the sub-area and tree topology, so we have to recall the Auto-discovery procedure to select a new root from sub-area and update the roots table.

- A tree node moved out sub-area: In this case, the moving tree node joins an existing sub-area or it can form a new sub-area by itself (tree node and root node) so in this case it effect on all tree.

- A root node moved out sub-area: when root node moved out its sub-area it affects the tree topology, if it moved in an existing subarea then it joins as a tree node, so the rest of its sub-area nodes recall Auto-discovery procedure to select a new root and maybe no one of them compatible with the root arrangement then we have many new sub-areas.

Therefore, we assume that the root node is not moving and in a stable position to ensure the tree topology performance.

\section{4. conclusion}

In this paper, we treaded to create a dynamic sub-area tree infrastructure for MANET in a geographical area. We used a hierarchical procedure to divide the whole network into many geographical sub-area networks. In the same geographical sub-area, proactive routing method issued between the sub-area root node and its interconnect nodes. In addition, a reactive routing method is also used among all sub-area root nodes. As a result of the new sub-area tree infrastructure, we obtained extensible routing improvement for MANET. The communication in the sub-area tree avoids the flooding procedures and routing in the sub-area tree avoids the transmission collision to optimize the networks.

\section{References}

[1] T. Qiu, N. Chen, K. Li, D. Qiao, and Z. Fu, "Heterogeneous ad hoc networks: Architectures, advances and challenges," Ad Hoc Networks, vol. 55, pp. 143-152, 2017. https://doi.org/10.1016/j.adhoc.2016.11.001.

[2] X. Liu, Z. Li, P. Yang, and Y. Dong, "Information-centric mobile ad hoc networks and content routing: a survey," Ad Hoc Networks, vol. 58, pp. 255-268, 2017. https://doi.org/10.1016/j.adhoc.2016.04.005.

[3] K. N. Qureshi and A. H. Abdullah, "Study of Efficient Topology Based Routing Protocols for Vehicular Ad-Hoc Network Technology," World Applied Sciences Journal, vol. 23, pp. 656-663, 2013.

[4] K. N. Qureshi and H. Abdullah, "Topology based routing protocols for VANET and their comparison with MANET," Journal of Theoretical and Applied Information Technology, vol. 58, pp. 707-715, 2013.

[5] I. Ouafaa, L. Jalal, K. Salah-ddine, and E. H. Said, "The comparison study of hierarchical routing protocols for ad-hoc and wireless sensor networks: A literature survey," in Proceedings of the The International Conference on Engineering \& MIS 2015, 2015, p. 32. https://doi.org/10.1145/2832987.2833039.

[6] C. Jothikumar and R. Venkataraman, "A review of hierarchical routing protocol for wireless sensor network," Indian Journal of Science and Technology, vol. 9, 2016. https://doi.org/10.17485/ijst/2016/v9i32/76215.

[7] H. Megharbi and H. Kheddouci, "Distributed algorithms for Constructing and Maintaining a Spanning Tree in a Mobile Ad hoc Network," in First International Workshop on Managing Context Information in Mobile and Pervasive Environments, 2005, p. 100.

[8] L. Gui-kai, S. Chun-li, W. Gang, and W. Hong-jiang, "Subarea Tree Routing in multi-hop wireless ad hoc networks," in Communication Systems, 2008. ICCS 2008. 11th IEEE Singapore International Conference on, 2008, pp. 1695-1699. https://doi.org/10.1109/ICCS.2008.4737470.

[9] R. P. Selvam and V. Palanisamy, "Stable and flexible weight based clustering algorithm in mobile ad hoc networks," International Journal of Computer Science and Information Technologies, vol. 2, pp. 824-828, 2011.

[10] R. Alubady, M. Al-Samman, A. Habbal, S. Hassan, and S. Arif, "Performance analysis of reactive and proactive routing protocols in MANET," ARPN J. Eng. Appl. Sci, vol. 10, pp. 1468-1478, 2015.

[11] D. N. Patel, S. B. Patel, H. R. Kothadiya, P. D. Jethwa, and R. H. Jhaveri, "A survey of reactive routing protocols in MANET," ICICES2014, 2014. https://doi.org/10.1109/ICICES.2014.7033833.

[12] P. K. Pandey and G. Biswas, "Design and implementation of binary tree based proactive routing protocols for large MANETS," in Advances in Computer Science and Information Technology, ed: Springer, 2010, pp. 397-408. https://doi.org/10.1007/978-3-642-13577-4_35.

[13] B.-K. Kim and D.-S. Eom, "A proposal of B-tree based routing algorithm for monitoring systems with regular movements in MANETs," Wireless personal communications, vol. 73, pp. 517-534, 2013. https://doi.org/10.1007/s11277-013-1200-2.

[14] J. Loo, J. L. Mauri, and J. H. Ortiz, Mobile ad hoc networks: current status and future trends: CRC Press, 2016. https://doi.org/10.1201/b11447. 